\title{
Vaginose Bacteriana em Mulheres com Infertilidade e em Menopausadas
}

Bacterial Vaginosis In Menopausal Women and in Women with Infertility

Miriam da Silva Wanderley, Carlos Roberto de Resende Miranda, Marcelo Jorge Carneiro de Freitas André Ricardo Sousa Pessoa, Alexandre Lauand, Rony Mafra Lima

\section{RESUMO}

Objetivo: analisar a prevalência de vaginose bacteriana (VB) em mulheres inférteis e em menopausadas e os métodos mais comumente usados na prática clínica para o seu diagnóstico. Métodos: foram avaliadas retrospectivamente 104 pacientes na menopausa e 86 inférteis. A presença de corrimento vaginal caracteristico, $p H$ vaginal $>4,5$, teste das aminas (whiff test) positivo e achado de vaginose bacteriana à coloração da secreção pelo Gram foram considerados positivos. Foi estabelecido diagnóstico de VB quando 3 dos 4 critérios acima fossem satisfeitos.

Resultados: analisando os métodos diagnósticos separadamente observamos, entre as menopausadas, 29 pacientes com corrimento vaginal característico (28,1\%), 10 (9,6\%) com whiff test positivo, $68(65,4 \%)$ com $p H$ vaginal $>4,5$ e $34(32,7 \%)$ com teste do Gram positivo. Nas mulheres inférteis os resultados foram 20 (23,2\%), 13 (15, 1\%), 61 (70,9\%) e 26 (30,2\%), respectivamente. Ao analisarmos todos os critérios em conjunto, em 14 pacientes na menopausa (13,5\%) e em 15 inférteis $(17,4 \%)$ foi diagnosticada $V B$.

Conclusão: a prevalência de VB foi similar nos 2 grupos de pacientes. Além disso, todos os métodos diagnósticos devem ser utilizados a fim de não se sub ou super-diagnosticar essa patologia.

PALAVRAS-CHAVE: Corrimento vaginal. Infertilidade. Menopausa. Vaginose bacteriana.

\section{Introdução}

A vaginose bacteriana (VB) é considerada, atualmente, a infecção vaginal de maior prevalência em mulheres em idade reprodutiva ${ }^{1-3}$. Foi originalmente descrita por Gardner e Dukes $1955^{4}$ como uma vaginite não específica caracterizada por secreção vaginal acinzentada, de odor fétido, com $\mathrm{pH}$ mais elevado que o normal, e com minima inflamação local, tendo como agente causal a Gardnerella vaginalis. Desde então, a presença de organismos anaeróbicos como Bacteroides spp, Mobiluncus spp, Mycoplasma hominis,

Área de Ginecologia e Obstetrícia, Faculdade de Medicina da Universidade de Brasília.

Correspodência:

Miriam da Silva Wanderley

Área de Ginecologia e Obstetrícia

Universidade de Brasília

Caixa Postal: 4360

70919-970 - Brasilia - DF

Telefone: (61) 273-3907/307-2535

e-mail: miriamsw@unb.br
Staphylococcus spp e Streptococcus spp tem sido, junto com a Gardnerella, altamente associada com esta condição ${ }^{5,6}$.

De etiologia não definida ${ }^{2}$, a VB é conceituada hoje como uma alteração da flora vaginal em que os lactobacilos, normalmente predominantes, são substituídos por uma flora complexa abundante, dominada por bactérias anaeróbias estritas e facultativas ${ }^{6,7}$, podendo também ser observados padrões intermediários de flora vaginal em que os microrganismos anaeróbios e lactobacilos coexistem ${ }^{3}$.

O estudo de Priestley et al..$^{8}$ levanta dúvidas sobre o que deveria ser considerado flora vaginal normal, já que torna-se dificil interpretar o achado de VB em mulheres assintomáticas ou o achado de flora normal em mulheres sintomáticas. Também Caillouette et al. ${ }^{9}$ questionam se estas mulheres sem sintomas deveriam ser sempre tratadas, pois observaram, em seu trabalho, que a presença da Gardnerella na secreção vaginal antecedeu o achado de VB. 
No entanto, a associação da VB com corioamnionite, abortamento tardio, doença inflamatória pélvica pós-aborto, parto pré-termo, endometrite pós-cesárea ${ }^{10}$ e mais recentemente como fator de risco para infecção do trato genital superior $^{11}$ sugere que todas as pacientes deveriam ser tratadas, apesar de ainda não se ter certeza de que isso resultaria em menos casos de infecção ou de complicações ginecológicas ${ }^{11}$, ou se a erradicação da Gardnerella reduziria o número de mulheres que apresentam $\mathrm{VB}^{9}$.

Sua prevalência é bastante variável, podendo ser desde $10 \%$ em mulheres em idade reprodutiva na população geral ${ }^{12}$, até $50 \%$ em populações de alto risco para uma doença sexualmente transmissivel (DST) ${ }^{1}$. No entanto, estima-se que praticamente a metade das mulheres com esta condição sejam assintomáticas ${ }^{7}$, podendo apresentar início e resolução espontâneas ${ }^{3}$, o que a tornaria tanto mais comum quanto subdiagnosticada ${ }^{5}$. Em mulheres na menopausa, nas quais há elevação natural do $\mathrm{pH}$ vaginal devido ao hipoestrogenismo, a prevalência de VB ainda é mais controvertida.

Dada a grande variedade na prevalência da VB tanto em mulheres em idade reprodutiva como na menopausa e as controvérsias quanto a esta condição estar ou não relacionada à atividade sexual ou "status" hormonal das pacientes, este trabalho tem por objetivo analisar a prevalência de VB em mulheres menopausadas e em mulheres inférteis devido ao fator tubário e os métodos diagnósticos correntemente utilizados na prática clínica para diagnosticá-la.

\section{Pacientes e Métodos}

Este estudo foi conduzido de forma retrospectiva com obtenção de informações em 190 prontuários de pacientes que procuraram o Hospital Universitário de Brasília (HUB), pela primeira vez, no período de janeiro de 1999 a novembro de 2000. Foram avaliadas 104 pacientes do Ambulatório de Climatério do HUB (Grupo I) que estavam há, no mínimo, 1 ano em amenorréia e cujos níveis de estradiol plasmático eram $\leq 20 \mathrm{pg} / \mathrm{mL}$ (radioimunoensaio). No Grupo II avaliaram-se 86 pacientes do Ambulatório de Reprodução Humana do HUB em investigação para o fator tubário de infertilidade. O fator masculino e todos os outros fatores femininos para infertilidade já haviam sido previamente investigados e descartados.

Nenhuma das 190 pacientes avaliadas estava em uso de qualquer medicação hormonal ou antibiótica, tópica ou sistêmica, nos seis meses que antecederam o estudo, que pudesse alterar os resultados obtidos. Todas as pacientes deram seu consentimento informado para a realização dos exames, que seguem a rotina diagnóstica previamente estabelecida em nosso serviço. O trabalho foi aprovado pelo Comitê de Ética em Pesquisa em Seres Humanos da Faculdade de Medicina da Universidade de Brasília.

Todas as pacientes foram avaliadas clinicamente por meio de exame ginecológico padrão, constando de inspeção externa e exame especular. O achado de secreção vaginal acinzentada, homogênea, de odor fétido e a ausência concomitante de sinais inflamatórios foram considerados positivos para VB.

Durante o exame especular a aferição do $\mathrm{pH}$ vaginal foi realizada aplicando-se a fita apropriada, do Laboratório Merck-Sharp-Dome, com gradação de 3,8 a 5,4, diretamente na parede lateral da vagina, no seu terço superior. Considerou-se positivo quando o $\mathrm{pH}$ foi maior que 4,5 .

Ainda durante o exame especular foi coletada secreção vaginal do fundo de saco posterior, com haste flexivel com ponta de algodão, e realizados três esfregaços longitudinais unidirecionais em lâmina de vidro para microscopia, sobre a qual foi feito o whiff test, o qual consiste em adicionar uma gota de $\mathrm{KOH} 10 \%$ ao fluido vaginal obtido. O desprendimento de odor de peixe podre caracteriza o teste positivo. Outra lâmina com a secreção obtida foi reservada para realizar análise do Gram.

Os esfregaços da secreção vaginal corada pelo Gram foram examinados em microscópio óptico com a objetiva de $100 \mathrm{X}$ de imersão, iniciando-se a leitura onde havia maior celularidade, e avaliados de acordo com a classificação de Nugent et al. ${ }^{13}$ (Figura 1). Considerou-se positivo quando a secreção obtida foi compativel com os graus $2 \mathrm{e}$ 3 desta classificação ${ }^{8,14}$.

\begin{tabular}{ll}
\hline Grau 1- Padrão normal & Predomínio de lactobacilos e poucos \\
& outros morfotipos bacterianos \\
& identificáveis (Gardnerella vaginalis, \\
& anaeróbios, flora Gram negativa, \\
& cocos Gram positivos)
\end{tabular}

Grau 2- Padrão intermediário Redução de lactobacilos e aumento de outros morfotipos

Grau 3- Vaginose bacteriana Ausência de lactobacilos e grande aumento no número dos outros morfotipos

Figura 1 - Classificação da flora vaginal de acordo com os critérios de Nugent et al. ${ }^{13}$ (simplificado). 
O diagnóstico final de VB foi estabelecido quando 3 dos 4 critérios acima foram satisfeitos ${ }^{15}$.

A comparação da prevalência de VB entre os dois grupos de pacientes foi feita por meio do teste do $\chi^{2}$. Considerou-se significativo quando $\mathrm{p}<0,05$.

\section{Resultados}

Em 14 pacientes menopausadas (13,5\%) foi estabelecido o diagnóstico de VB. A média de idade dessas pacientes foi de 52 anos, sendo que 78 eram casadas e referiam relações sexuais em intervalos que variavam de 1 vez por semana até bastante esporádicas. Todas referiam parceiro único há mais de 10 anos. As outras 26 pacientes, viúvas ou solteiras, negavam relacionamentos sexuais há mais de 2 anos, em média. Todas as pacientes com VB eram casadas e somente duas referiam ter sido tratadas por recomendação do médico do parceiro, mas desconheciam o diagnóstico (Tabela 1).

Tabela 1 - Características clínicas das pacientes estudadas nos dois grupos.

\begin{tabular}{lcc}
\hline & Menopausadas & Inférteis \\
\hline $\begin{array}{l}\text { Número de Pacientes } \\
\text { Idade média }\end{array}$ & 104 & 86 \\
Estado civil & 52 anos & 30,5 \\
$\quad$ casada & 78 & 80 \\
$\quad$ viúva & 10 & - \\
$\quad$ união estável & - & 6 \\
$\quad$ solteira & 16 & - \\
Freqüência das & desde 1 vez/semana & $3-4 v e z e s /$ semana \\
relações sexuais & até esporádicas & \\
No de parceiros sexuais nos & 1 & 2,1 \\
últimos 5 anos (média) & & 8 \\
DST & & 7 \\
prévia conhecida e tratada & - & 71 \\
trattada sem diagnóstico & 2 & \\
desconheciam ou negavam & 102 & \\
\hline
\end{tabular}

No grupo I, apesar de $38(36,5 \%)$ pacientes referirem corrimento vaginal à consulta ginecológica, somente $28,1 \%$ delas o apresentavam com as características clínicas de VB. O $\mathrm{pH}$ vaginal foi maior que $4,5 \mathrm{em} 65,4 \%$ das pacientes e o whiff test revelou-se positivo em 9,6\% delas. O teste do Gram apresentou resultado positivo em 32,7\% das pacientes. Quando avaliamos todos os critérios diagnósticos em conjunto, observamos efetivamente VB em 14 pacientes $(13,5 \%)$.

Nas mulheres com infertilidade por fator tubário o diagnóstico de VB foi estabelecido em 15 delas $(17,4 \%)$. A idade média destas pacientes foi de 30,5 anos, sendo que 80 eram casadas e 6 referiam união estável há mais de 2 anos. Oito pacientes referiam DST previamente, 7 não souberam referir se haviam tido ou não uma DST, mas haviam feito algum tipo de tratamento por recomendação do médico de seu parceiro, e 71 negavam ou desconheciam qualquer doença de transmissão sexual (Tabela 1).

Quanto aos critérios diagnósticos para VB no Grupo II, 31 pacientes (36\%) apresentaram queixa clínica de corrimento vaginal, mas somente em $23,2 \%$ delas foi observada a secreção vaginal característica. O whiff test revelou-se positivo em $15,1 \%$ e o $\mathrm{pH}$ vaginal foi maior que 4,5 em $70,9 \%$ das pacientes. A coloração das lâminas pelo Gram foi positiva em 26 casos (30,2\%). Avaliando-se os 4 critérios juntos, diagnosticouse VB em $17,4 \%$ das pacientes inférteis por fator tubário.

Não observamos diferença estatisticamente significativa entre a prevalência de VB em mulheres menopausadas $(13,5 \%)$ e com infertilidade por fator tubário $(17,4 \%)\left(\mathrm{p}>0,05 ; \chi^{2}\right)(\mathrm{Ta}-$ bela 2; Figura 2).

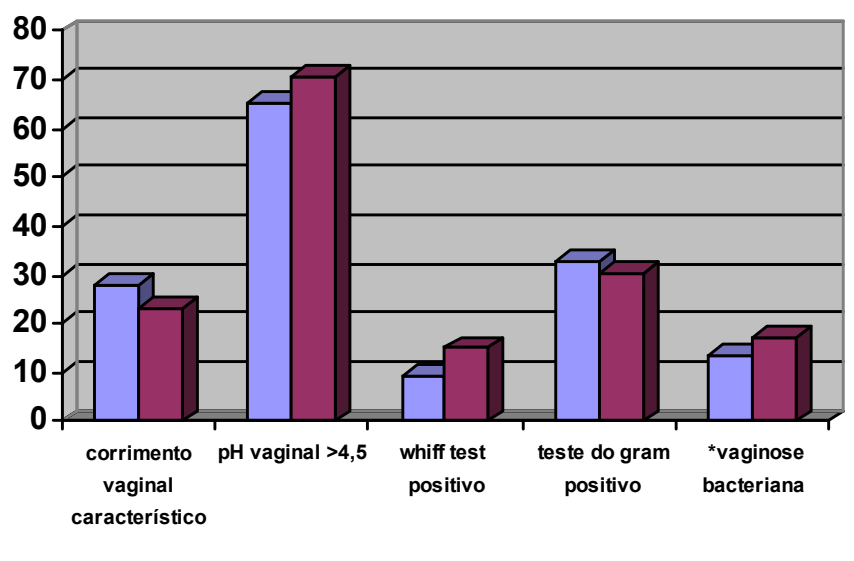

$\square$ Pacientes menopausadas $\square$ Pacientes Inférteis por fator tubário

* $p>0,05, \chi^{2}$ para o diagnóstico final de vaginose bacteriana.

Figura 2 - Critérios diagnósticos e diagnóstico final de vaginose bacteriana em mulheres menopausadas e inférteis. 
Tabela 2 - Critérios diagnósticos e diagnóstico final de vaginose bacteriana em mulheres menopausadas (grupo I) e inférteis (grupo II).

\begin{tabular}{lcccc}
\hline Critérios diagnósticos & \multicolumn{2}{c}{ Grupo I (Menopausadas) } & Grupo II (Inférteis) & \% \\
\hline Corrimento vaginal típico & $\mathbf{n}$ & $\%$ & 20 & 23,2 \\
pH vaginal $>$ 4,5 & 29 & 28,1 & 61 & 70,9 \\
Whifftest & 68 & 65,4 & 13 & 15,1 \\
Teste do Gram & 10 & 9,6 & 26 & 30,2 \\
Vaginose bacteriana & 34 & 32,7 & 15 & $17,4^{*}$ \\
(Diagnóstico final) & 14 & $3,5^{*}$ & & \\
\hline
\end{tabular}

${ }^{*} p>0,05, \chi^{2}$ para o diagnóstico de vaginose bacteriana.

\section{Discussão}

A vaginose bacteriana é caracterizada microbiologicamente por uma mudança na flora vaginal, na qual flora dominante composta por lactobacilos é substituída por outra, mista, que inclui Gardnerella vaginalis, Bacteroides spp, Mobiluncus spp e Mycoplasma hominis ${ }^{5,6}$. Sua prevalência é bastante variável, podendo ser de $11 \%^{16}$ na população geral até $63 \%$ em clínicas de $\mathrm{DST}^{1}$. E a sua conotação exclusivamente sexual tem sido questionada por alguns autores ${ }^{17}$, já que a presença de VB tem sido observada em mulheres assintomáticas $^{18}$, lésbicas ${ }^{19}$ e em adolescentes virgens ${ }^{20}$.

Em nosso trabalho observamos uma prevalência de VB de $13,5 \%$ em mulheres na menopausa e de $17,4 \%$ em pacientes inférteis, diferença essa não significativa, apesar de estas referirem relações sexuais desprotegidas com muito maior freqüência que aquelas. No entanto, ambos os grupos referiram estar com o mesmo parceiro há, no mínimo, 2 anos. Lactobacilos e bactérias associadas à VB são menos comumente parte da microflora vaginal em mulheres na pós-menopausa do que em mulheres em idade reprodutiva ${ }^{21}$, o que poderia explicar a menor prevalência de VB que observamos no Grupo I, mais do que a freqüência de relações sexuais desprotegidas ou o número de parceiros que as pacientes referiram.

$\mathrm{O}$ fato de diversos autores ${ }^{3,22}$ terem observado modificação no microambiente vaginal na fase proliferativa do ciclo, e conseqüentemente maior predisposição à VB recorrente nessa fase, sugere que a perda da barreira cervical durante a menstruação ou modificações cíclicas hormonais ${ }^{22}$ poderiam estar ligadas à patogênese da VB. Apesar de Nilsson et al. ${ }^{23}$ terem observado que a VB está associada com comportamento sexual de risco, similar ao encontrado na infecção por clamídia, nossos resultados suportam a visão de que a VB também pode ser secundária a um pro- blema metabólico subjacente ${ }^{17}$, e não sempre secundário a uma DST ou a promiscuidade sexual, apesar de nenhuma das pacientes menopausadas, que não tinham mais relações sexuais, terem apresentado VB.

A falta de entendimento sobre o início da VB, sobre uma possivel resolução espontânea, das recorrências sintomáticas ${ }^{24}$, do achado de que algumas mulheres parecem ter VB de forma mais ou menos constante, ao passo que outras parecem ser resistentes a esta condição ${ }^{10}$, da presença da Gardnerella na secreção vaginal de mulheres assintomáticas ${ }^{14}$, fazem com que o manejo clínico dessas pacientes nem sempre seja adequado e o tratamento efetivo.

A primeira técnica padronizada para diagnosticar VB foi proposta por Amsel et al. ${ }^{15}$ e é baseada em um conjunto de critérios clínicos, já que esta não é causada por um único agente, mas uma condição polimicrobiana ${ }^{1,13}$. Schwebke et al. ${ }^{1}$ afirmam que a natureza subjetiva inerente na avaliação dos critérios clínicos pode resultar em significativo subdiagnóstico da VB em alguns centros. Apesar da baixa prevalência observada em nosso estudo, esta foi semelhante à encontrada em trabalhos na literatura ${ }^{12,16}$. O fato de termos utilizado a associação de métodos diagnósticos para VB conforme proposição de Amsel et al. ${ }^{15}$ e não um único método isolado afasta a possibilidade de subdiagnóstico e reforça a acurácia dos nossos resultados.

Um conceito amplamente divulgado é que a vaginose é uma condição bastante comum, que causa um corrimento vaginal com típico odor de peixe $^{24}$. Sendo assim, seria de esperar que todas as pacientes apresentassem essas características clínicas ao exame, o que não observamos em nosso trabalho. Apesar de mais de um quarto das pacientes, de ambos os grupos, terem referido corrimento vaginal sugestivo de $\mathrm{VB}$, a observação clínica confirmou o diagnóstico em $28,1 \%$ das pacientes do Grupo I e 23,2\% do Grupo II. No entanto, a presença objetiva do corrimento típico, observado 
pelo médico, esteve sempre associado com o diagnóstico final de VB, semelhante ao estudo de $\mathrm{Hay}^{24}$. Quanto ao whiff test, foi positivo em $10(9,6 \%)$ mulheres menopausadas e em $13(15,1 \%)$ inférteis. Segundo Blackwell et al. ${ }^{25}$, parece que o componente anaeróbico, mais do que a Gardnerella, é responsável pelo teste das aminas positivo, teste este que depende do volume da secreção vaginal e da concentração das aminas nesta secreção ${ }^{5}$. Assim, os resultados falso-negativos que observamos em nosso estudo poderiam ser explicados pela presença de um corrimento profuso com baixa concentração de aminas ou vice-versa.

Os estudos da literatura ${ }^{9,10}$ indicam que um $\mathrm{pH}$ vaginal menor que 4,5 é boa indicação de que a paciente não tem VB e de que mantém bons níveis estrogênicos. $\mathrm{O}$ achado de $\mathrm{pH}$ vaginal elevado $(5,0-6,5)$ em pacientes normalmente estrogenizadas está quase sempre associado à $\mathrm{VB}^{26}$. A vagina na pré-puberdade e na pós-menopausa possui epitélio atrófico com $\mathrm{pH}$ normal de superfície de $6,0-8,0^{9}$. Dessa forma, não é surpresa que a porcentagem de resultados falso-positivos nas pacientes menopausadas tenha sido elevada, especialmente porque nenhuma delas estava em uso de terapia de reposição hormonal. Já as inférteis, com ciclos regulares, também apresentaram $\mathrm{pH}$ elevado em uma porcentagem anormalmente alta $(70,9 \%)$ de casos, que não poderiam ser atribuídos a uma deficiência estrogênica, nem a uma infecção concomitante por tricomoniase, que também poderia elevar o $\mathrm{pH}$, mas outras causas como infecções não-anaeróbicas, irritantes ou alergenos locais e problemas dermatológicos deveriam ser $\operatorname{considerados}^{27}$. No entanto, não observamos nenhum resultado falso-negativo em ambos os grupos, o que reforça o fato de o $\mathrm{pH}$ vaginal ser um método diagnóstico útil, além de simples e barato, concordando com diversos trabalhos na literatu$\mathrm{ra}^{9,28}$.

A importância do diagnóstico de VB tem sido enfatizada pela observação da associação desta com vários problemas obstétricos e seqüelas ginecológicas ${ }^{5,23}$. Paavonen et al. ${ }^{29}$ e Eschenbach et al. ${ }^{30}$ observaram aumento no diagnóstico clínico de doença inflamatória pélvica em mulheres com VB. Contudo, Peipert et al. ${ }^{11}$ não conseguiram estabelecer relação temporal efetiva entre ambas as condições, permanecendo a dúvida se o tratamento indiscriminado da VB, em mulheres sintomáticas e assintomáticas, resultaria em menor porcentagem de seqüelas reprodutivas subseqüentes. Em nosso estudo, em que somente 15 das 86 pacientes com infertilidade de causa tubária tiveram VB, não foi possível estabelecer uma associação causa-efeito entre essas duas condições.
Dessa forma, podemos concluir que, apesar de termos observado maior prevalência de VB em mulheres em idade reprodutiva, essa não foi significativamente mais elevada do que em mulheres na menopausa, o que nos leva a especular que outras condições externas tais como duchas vaginais e irritantes locais, o meio ambiente vaginal, variável de paciente para paciente, resistência individual às modificações da microflora local e alterações metabólicas outras tenham influência mais significativa e decisiva sobre o aparecimento da VB do que a transmissão sexual desta condição em si. Além disso, todos os critérios clínicos deveriam ser utilizados no diagnóstico da VB, a fim de se evitar sub ou superdiagnosticar essa doença.

\section{SUMMARY}

Purpose: to evaluate the prevalence of bacterial vaginosis $(B V)$ in menopausal and in infertile outpatients and to analyze the current clinical diagnostic methods.

Methods: we evaluated retrospectively 104 menopausal women and 86 with infertility. Characteristic vaginal discharge on gynecological examination, $p H>4.5$, positive $\mathrm{KOH}$ whiff test, and bacterial vaginosis by Gram test were considered positive. $B V$ was established when at least 3 out of 4 criteria were found.

Results: among the menopausal women, 29 patients (28.1\%) were clinically positive for $B V, 10$ (9.6\%) had positive whiff test, 68 (65.4\%) vaginal $p H>4.5$, and 34 (32.7\%) positive Gram test. For the infertile patients the figures were 20 (23.2\%), 13 (15.1\%), 61 (70.9\%) and $26(30.2 \%)$, respectively. According to our established criteria, $B V$ was diagnosed in 14 menopausal (13.5\%) and 15 infertile (17.4\%) women.

Conclusion: bacterial vaginosis prevalence was similar in both groups of patients. In addition, all diagnostic criteria should be followed in order to avoid underdiagnosing this pathology or treating an otherwise normal vaginal flora.

KEY WORDS: Vaginal discharge. Infertility. Menopause. Bacterial vaginosis.

\section{Referências}

1. Schwebke JR, Hillier SL, Sobel JD, McGregor JA, Sweet RL. Validity of the vaginal gram stain for the diagnosis of bacterial vaginosis. Obstet Gynecol 1996; 88:573-6. 
2. Chaim W, Mazor M, Leiberman JR. The relationship between bacterial vaginosis and preterm birth. A review. Arch Gynecol Obstet 1997; 259:51-8.

3. Hay PE, Ugwumadu A, Chowns J. Sex, thrush and bacterial vaginosis. Int J STD AIDS 1997; 8:603-8.

4. Gardner HL, Dukes CD. Haemophilus vaginalis vaginitis: a newly defined specific infection previously classified as non-specific vaginitis. Am J Obstet Gynecol 1955; 69:962-76.

5. O'Dowd TC, West RR, Winterburn PJ, Hewlins MJ. Evaluation of a rapid diagnostic test for bacterial vaginosis. Br J Obstet Gynaecol 1996; 103:366-70.

6. Frega A, Stentella P, Spera G, et al. Cervical intraepithelial neoplasia and bacterial vaginosis: correlation or risk factor? Eur J Gynaecol Oncol 1997; 18:76-7.

7. Priestley CJ, Kinghorn GR. Bacterial vaginosis. Br J Clin Pract 1996; 50:331-4.

8. Priestley CJ, Jones BM, Dhar J, Goodwin L. What is normal vaginal flora? Genitourin Med 1997; 73:23-8.

9. Caillouette JC, Sharp CF Jr, Zimmerman GJ, Roy S. Vaginal $\mathrm{pH}$ as a marker for bacterial pathogens and menopausal status. Am J Obstet Gynecol 1997; 176:1270-7.

10.Platz-Christensen JJ, Pernevi P, Hagmar B, Andersson E, Brandberg A, Wiqvist N. A longitudinal follow-up of bacterial vaginosis during pregnancy. Acta Obstet Gynecol Scand 1993; 72:99-102.

11.Peipert JF, Montagno AB, Cooper AS, Sung CJ. Bacterial vaginosis as a risk factor for upper genital tract infection. Am J Obstet Gynecol 1997; 177:1184-7.

12.Morgan DJ, Aboud CJ, McCaffrey IM, Bhide SA, Lamont RF, Taylor-Robinson D. Comparison of Gram-stained smears prepared from blind vaginal swabs with those obtained at speculum examination for the assessment of vaginal flora. Br J Obstet Gynaecol 1996; 103:1105-8.

13.Nugent RP, Krohn MA, Hillier SL. Reliability of diagnosing bacterial vaginosis is improved by a standardized method of gram stain interpretation. J Clin Microbiol 1991; 29:297-301.

14. Hillier SL. Diagnostic microbiology of bacterial vaginosis. Am J Obstet Gynecol 1993; 169:455-9.

15.Amsel R, Totten PA, Spiegel CA, Chen KC, Eschenbach D, Holmes KK. Nonspecific vaginitis. Diagnostic criteria and microbial and epidemiologic associations. Am J Med 1983; 74:14-22.

16.Hay PE, Taylor-Robinson D, Lamont RF. Diagnosis of bacterial vaginosis in a gynaecology clinic. $\mathrm{Br}$ J Obstet Gynaecol 1992; 99:63-6.

17.Brand JM, Galask RP. Trimethylamine: the substance mainly responsible for the fishy odor often associated with bacterial vaginosis. Obstet Gynecol 1986; 68:682-5.
18.Bump RC, Zuspan FP, Buesching WJ $3^{\text {rd }}$, Ayers LW, Stephens TJ. The prevalence, six-month persistence, and predictive values of laboratory indicators of bacterial vaginosis (nonspecific vaginitis) in asymptomatic women. Am J Obstet Gynecol 1984; 150:917-24.

19.Skinner CJ, Stokes J, Kirlew Y, Kavanagh J, Forster GE. A case-controlled study of the sexual health needs of lesbians. Genitourin Med 1996; 72:27780.

20.Bump RC, Buesching WJ $3^{\text {rd }}$. Bacterial vaginosis in virginal and sexually active adolescent females: evidence against exclusive sexual transmission. Am J Obstet Gynecol 1988; 158:935-9.

21.Hillier SL, Lau RJ. Vaginal microflora in postmenopausal women who have not received estrogen replacement therapy. Clin Infect Dis 1997; 25 Suppl 2:S123-6.

22.Korn AP, Hessol NA, Padian NS, et al. Risk factors for plasma cell endometritis among women with cervical Neisseria gonorrhoeae, cervical Chlamydia trachomatis, or bacterial vaginosis. Am J Obstet Gynecol 1998; 178:987-90.

23.Nilsson U, Hellberg D, Shoubnikova M, Nilsson S, Mardh PA. Sexual behavior risk factors associated with bacterial vaginosis and Chlamydia trachomatis infection. Sex Transm Dis 1997; 24:241-6.

24. Hay PE. Recurrent bacterial vaginosis. Dermatol Clin 1998; 16:769-73, xii-xiii.

25.Blackwell AL, Fox AR, Phillips I, Barlow D. Anaerobic vaginosis (non-specific vaginitis): clinical, microbiological, and therapeutic findings. Lancet 1983; 17:1379-82.

26. Hillier SL, Nugent RP, Eschenbach DA, et al. Association between bacterial vaginosis and preterm delivery of a low-birth-weight infant. $\mathrm{N}$ Engl J Med 1995; 333:1737-42.

27.Spinillo A, Bernuzzi AM, Cevini C, Gulminetti R, Luzi S, De Santolo A. The relationship of bacterial vaginosis, Candida and Trichomonas infection to symptomatic vaginitis in postmenopausal women attending a vaginitis clinic. Maturitas 1997; 27:253-60.

28.Krohn MA, Hillier SL, Eschenbach DA. Comparison of methods for diagnosing bacterial vaginosis among pregnant women. J Clin Microbiol 1989; 27:1266-71.

29.Paavonen J, Teisala K, Heinonen PK, et al. Microbiological and histopathological findings in acute pelvic inflammatory disease. $\mathrm{Br} \mathrm{J}$ Obstet Gynaecol 1987; 94:454-60.

30.Eschenbach DA, Hillier SL, Critchlow CW, Stevens C, DeRouen T, Holmes KK. Diagnosis and clinical manifestations of bacterial vaginosis. Am J Obstet Gynecol 1988; 158:819-28. 